\title{
ABBREVIATED ENGLISH - A TYPICAL FEATURE OF ONLINE COMMUNICATION?
}

Keywords: English, abbreviations, CMC, Social Networking Sites, gender

\begin{abstract}
Since electronic communication became widespread, one of the typical features identified in e-language and commented on, also as an aspect of linguistic analyses, has been acronyms and abbreviated language of various kinds. It has been particularly visible in English, as this language, unlike many others, shows great flexibility with regard to such modifications, mainly due to homophony between numerous words and individual sounds as well as lack of inflectional endings, which otherwise would limit the abbreviation options. However, the current overview of a selection of social networking sites does not appear to demonstrate any striking presence of this marker of computer-mediated communication (CMC). The present analysis therefore attempts to investigate the contemporary status of online language abbreviations in English, with the aim of discovering the actual visibility and frequency of use of such items as well as identifying their most popular forms found online. In particular, the research focuses on three social networking sites, i.e. Facebook (private accounts and fanpages), YouTube, and Twitter, trying to establish which factors contribute to the preference for abbreviations: the limitation of the post length, the degree of informality and closeness to the post addressees or the anonymity of the post author. Additionally, the investigation also takes into account the nationality of the users, and notably the status of English as their first or second language, as well as their gender, on the assumption that these variables play a significant role in the selection of this aspect of online discourse.
\end{abstract}

\section{Introduction}

Almost since the time when online communication became widely accessible, and especially since the introduction of online discussion lists and social networking sites linguists have been paying attention to features of online language that make 
it different from offline communication. Apart from focusing on the central issue of hybridization of Internet communication, spanning the heretofore speech vs. writing hiatus, at least in the formalized and semi-formalized context, what has constantly attracted scholars' attention has been the unique orthographic code that has developed alongside, a characteristic Netlingo, as some call it (cf. Danesi 2016). Suffice to say, the majority of observations have concerned the English language, especially in the early days of the Internet, when English occupied ca. $85 \%$ of online resources (cf. Graddol 2006). The classification of Netlingo types was presented, among others, by Crystal (2001, 2006, 2011), Baron (2008), Thurlow, Lengel, Tomic (2004), and, as mobile telephony became the technology of everyday use, it also included the analysis of text messages (cf. Crystal 2008; Anis 2007; Dąbrowska 2011). With time, other languages began to adapt to the new conventions as well, and respective analyses of the forms and frequencies of use followed (cf. Ling 2005 on Norwegian; Palfreyman, Al Khalil 2007 on Arabic; Su 2007 on Taiwanese Chinese; Anis 2007 on French; Dąbrowska 2011 on Polish vs. English; Bieswanger 2007, 2010 on German vs. English; Dąbrowska 2012 on Indian English).

\section{Overview of the problem}

The current analysis has been motivated by some more recent discussion concerning the use of the Internet code in English (cf. Deumert 2014; Danesi 2016), and notably by somewhat puzzling claims concerning the almost obligatory use of abbreviated language online. Deumert (2014: 130) states that "the imperative to deviate from normative standard remains an important maxim of informal digital writing" and refers to boyd's (2008: 129) conclusions that such informal spellings constitute "markers of cool" and allow the writers to "dramatise" their online communication. Likewise, following McIntosh's (2010, cited in Deumert 2014: 130) analysis of texting strategies used by Kenyans in English, which she calls "a global English medialect," it may be concluded that not only the use of modern technologies, but also of the code that goes with them makes people act "modern". Also Danesi (2016:67) maintains that the level of formality online is "determined by whether or not abbreviations are used," and claims that the use of some linguistic strategies like $u$ instead of you in text messages is the expected form, for the standard spelling may carry some additional meaning, including undertones of anger or irony. Similarly, some of the research adduced by Deumert suggests that using the full standard spelling of words may indicate that the author is serious about what s/he is saying or wants to convey respect towards the addressee, as e.g. when declaring one's love to someone, according to the principle that "if you mean it make an effort." Incidentally, unusual brevity may at times mark the author's negative emotions too and indicate that something is actually wrong (Deumert 2014: 139).

The above comments allow us to conclude that the use of abbreviations in Internet communication has acquired a different meaning than the abbreviations that were used in writing, both ancient manuscripts and later printed texts, in the 
pre-electronic era. Shortenings of texts of different kinds are by no means a thing of the present-day use of language. As early as ancient times Marcus Tullius Tiro is believed to have developed some form of shorthand to take notes of Cicero's speeches (Danesi 2016: 67). Likewise, Old English and Middle English scribes made use of some abbreviated forms, e.g. the symbol resembling the figure 7 to indicate and, or deletion of letters in frequently used words, e.g. sci for sancti or $\bar{e}$ for est (cf. Freeborn 1998: 30-31). With the advance of print Renaissance and Enlightenment writers made frequent use of superscripts applied especially in common function words, notably pronouns, prepositions, etc., e.g. $w^{t}$ hono ${ }^{r}$ for with honour or $S^{r}$ for Sir (Freeborn 1998: 274, 278). Modern scholarly texts customarily utilize certain Latin abbreviations as well, e.g. NB, et al., etc. It may thus be deduced that in the non$\mathrm{CMC}$ use of language the primary reason for the development of such a code has been to facilitate note-taking and speed up written communication (cf. the use of shorthand in court hearings). Along with this, however, a certain conventionality has developed, which, as in the case of academic text abbreviations, or specialized registers (Seaspeak, aviation English, CB radio jargon, etc.), has not only made communication more efficient as a time- and space-saving device (cf. Saenger 2010), but also begun to mark the in- and out-group membership of those involved in the communication process. When Netlingo is taken into consideration, it might be concluded that it is in fact the latter of the two reasons that has dominated Internet communication. Indeed, some research concerning the use of abbreviations in text messages in particular as well as in Tweets has demonstrated that text shortenings were and are in part a result of the limited number of characters allowed in the message (traditionally 160 for text messages and 140 for Tweets, with attempts to double the number for the latter as of September 2017) and act as hit-saving device (cf. Anis 2007; Dąbrowska 2011). The same is to an extent true of Instant Messaging, where the speed of writing is certainly at stake. Yet, as the analysis of some forms of online communication has proved, applying these forms of spelling actually makes the typing more demanding and time-consuming, as e.g. illustrated by the use of a very complicated combination of letters, capital and lower case, numbers and other characters by a Zambian user on IM (cf. Coetzee 2012: 111, cited in Deumert 2014: 135), the use of leetspeak, where letters are substituted by other characters on the basis the similarity of their look (cf. Deumert 2014), or else the application of Camel Case (known alternatively as Bumpy Case, bicapitalization, etc., as e.g. in YouTube, iPhone, cf. Crystal 2013). As Stevenson (2000 n.p., cited in Palfreyman, Al Khalil 2007: 46) claimed with regard to the phonetic spelling on Internet Relay Chat, it was not used because of a particular desire to reflect pronunciation in writing, but was rather "a result of social pressure to break conventional spelling rules and comply with IRC's non-conformist, hacker image."

It may therefore be assumed that, if not induced primarily by necessity, but fashion, the use of abbreviations in online communication, especially that of a more public character, could be of somewhat fleeting nature and on the whole not stand the test of time. This is the claim that will underlie the present analysis as regards the use of the English language, as the source of the greatest number of possibilities 
of language shortening, on social networking sites. The assumption thus is that, contrary to the claims quoted above about the pressure to use non-conventional spellings, CMC users will apply this mode of writing relatively infrequently, and, as will be demonstrated, also to a varying degree as regards various types of Social Networking Sites (SNSs).

Further to this point, as some studies have demonstrated, even English alone, despite its exceptional properties rendering the language especially prone to shortenings of various kinds due to its high sound-word homophony and very limited number of inflections, which as a result keeps the form of words fairly stable, may show a considerable variation in the way it is spelled depending on where it is used, notably countries where it is the native language, as opposed to where it is the second language. For instance, studies concerning the use of the pronoun you in full or in its $u$ form conducted in the US (Squires 2010), Canada (Tagliamonte, Denis 2008), the UK (Tagg 2009), and South Africa, Ghana and Nigeria (Deumert, Lexander 2013) with regard to its use in Instant Messaging (investigated in the US and Canada studies) and in text messages (analyzed by the remaining ones) clearly demonstrated that tendencies of using the abbreviated form there vary enormously, stretching from barely $7.4 \%$ in the US to as much as $88 \%$ in Nigeria (cf. Deumert 2014: 141). There is then no single global norm that all English-speaking users follow, especially that, as Danesi (2016) stresses, the online standard is not regulated by any official language policy in the first place. Deumert (2014: 141; cf. Pennycook 2007; Blommaert 2010) points out that certain uses and linguistic strategies become local practices, with local indexicalities of various forms. The local practice is, no doubt, linked with the perception of a given language or languages (notably in multilingual settings) by the given speech community. Some investigation of local practices in multilingual settings has for instance reported a certain duality in texts written with the help of two languages relevant for the analyzed community, e.g. German-Turkish in Germany (cf. Hinnenkamp 2008) or English-isiXhosa in South Africa (Deumert 2014). In the texts analyzed one of the tongues - the most dominant one locally (i.e. German and English, respectively, in the contexts investigated) tended to be orthographically manipulated with, as opposed to the other - the less dominant, minority languages, which were written in their standard form. As one of the respondents, from South Africa, elucidated, she tended to modify English as the dominant tongue, for she had no respect for it, while the altering of isiXhosa, which she identified with more, would appear very inappropriate to her (Deumert 2014: 137). Such a negative perception of the socially and politically powerful tongues cannot be considered universal, however, as studies of some other pairs of languages have shown, it is certainly worth taking it into consideration when analyzing various online practices and interpreting them. It may be suspected, though, that when such a pairing takes place with the presence of English as one of the tongues, abbreviating English can be observed in online communication to a considerable extent, for, as Agha (2005, cited in Deumert 2014: 138) has suggested, English, by contrast to the more locally limited tongues, is a modern voice, cool, and therefore does not need to be recorded correctly, as opposed to the local tongues, which are rooted in tradition and as such call for greater 
respect. The fact that English has become a language of almost everyone may lead to a belief that it does not index any particular set of cultural values, contrary to less socially dominant languages. This, in turn, may encourage its users to mould it according to their local perception and attitudes they hold towards it. The material analyzed below reflects such differences too - one can compare posts like, e.g. Imao faye started that and was nowhere to be found Imaooo used on YouTube by a female native English speaker, with Thx IndiaToday for the platform. Had a great time \& happy 2 wear a suit for $\boldsymbol{u}$. Mr.Puri \& team nxt time dinner fr sure posted by a male Indian celebrity on Twitter in order to appreciate the different preferences of users depending on their culture, gender, and the medium of expression used.

\section{Analysis of data}

In view of the above the following analysis will focus on samples of English excerpted from three currently most popular and internationally widespread Social Networking Sites, i.e. Facebook, YouTube and Twitter, which attract an international membership, and whose language of communication is frequently English. Thus, they are publicly accessible sites whose main purpose (especially Facebook and YouTube), as various studies have demonstrated, is to maintain contacts with others, create a positive social image (cf. "social grooming"), and exchange news. This also concerns Twitter, although the official purpose of the site, as its creators claimed, was meant to be news updates (cf. Danesi 2016: 108). Such a context of communication, in some of the sites additionally enhanced by anonymity of the users, appears to be the right kind of a milieu in which informal language can be used.

More specifically, the analysis will be based on the following data:

\section{- YouTube}

- 'Moonlight' or 'La La Land'? Best Picture Mix-up at Oscars (7o posts - women 35, men 35)

- FAN | Official Trailer|Shah Rukh Khan (7o posts - women 35, men 35)

\section{- Twitter}

- Twitter celebrity profiles (US and UK): Jennifer Lawrence, Keira Knightley, Queen Latifah, Emma Watson, Rihanna, Madonna, Selena Gomez, Nicholas Hoult, Tom Hollander, Brad Pitt, Leonardo di Caprio, Chris Rock, Snoop Dog, Justin Bieber (70 posts - women 35, men 35) ${ }^{1}$

- Twitter celebrity profiles (India): Priyanka Chopra, Deepika Padukone, Alia Bhatt, Kajol, Anushka Sharma, Shah Rukh Khan, Aamir Khan, Ranbir Kapoor, Karan Johar, Deepak Ajwani (7o posts - women 35, men 35)

Both Rihanna (born and raised in Barbados) and Justin Bieber (born and raised in Ontario, Canada) have been classified as members of this group due to the fact that they have now been long linked with the US both professionally and privately; what is more, both Barbados and Canada use English as their first language too. 


\section{- Facebook fanpage}

- Facebook fanpage: Oscars 2017 (posts from 27 February, 2017) - 70 posts (women 35, men 35)

- Facebook fanpage: Fan (posts from Fabruary-March 2017) - 70 posts (women 35, men 35)

\section{- Facebook private profiles}

- Facebook private profiles: British and American users (7 women, 7 men) 70 posts (women 35 , men 35 )

- Facebook private profiles: Indian users (7 women, 7 men) - 70 posts (women 35, men 35)

The discussions selected from YouTube and Facebook fanpages concern the ceremony of the Oscar Academy Awards 2017, notably the erroneous announcement of the winner in the best picture category, and parallel to it a discussion of the Indian film Fan is analyzed, on the assumption that emotionally loaded comments would lead to a greater informality of the language, which could, in turn, manifest itself also in non-standard spelling of words. The analysis of these highly informal displays of language, not infrequently paired up with the anonymity of the users, is then juxtaposed with Facebook personal posts collected from private accounts of British and American Facebook users and a parallel group of Indian Facebook users, with the additional aim of further investigating possible local approaches to the use of English as the native and second language, respectively. There the profile owners communicate with people they mostly know, also from the offline world, as a result of which their language should assume a more polite-to-neutral character. The sample is completed with Twitter messages collected from a group of female and male British and American as well as Indian celebrities (movie stars and singers). In the latter group the users are then known, much as in the case of Facebook private accounts, yet, as might be assumed, it is mostly one-sided familiarity. At the same time the intention of the post authors may rather be the need to win acceptance and popularity, which in turn may manifest itself in some non-standard behaviour, including the use of language too. As seen above, each category is illustrated by an equal number of posts written by women and men, respectively, so that there is a possibility to compare the linguistic strategies found in the analyzed posts in respect of the users' gender. Thus, the total number of posts analyzed for the purpose of the study is 560 , representing 280 written by women and $28 \mathrm{o}$ by men, and at the same time 280 posts written by Brits and Americans, and 280 by Indian users.

As the initial overview of the material has demonstrated, the actual object of investigation will be acronyms, lower case spelling, sound-word substitutions, apostrophe omission, clippings, vowel deletions, phonetic spellings, number-word substitutions and rebus writing, with the aim of establishing whether any of these have over the recent years gained a particular popularity among the analyzed groups of users, and whether any of them have indeed become an almost mandatory element of online communication and on which of the sites. 


\section{The medium}

The first step of the analysis is the most general presentation of the data in terms of the medium of communication. The scanning of the 560 posts $(10,195$ words in all) has generated 251 examples of abbreviated English in total ( 0.45 item per post, $2.5 \%$ of all words used in the posts). When divided into the four types of platforms, the distribution presents itself as follows:

- YouTube (3850 words) - 110 items, i.e. $2.8 \%$ of all the words on YouTube (44 used by native speakers in 2407 words, i.e. $1.8 \%$ of all the words +66 utilised by Indian speakers in 1443 words in all, i.e. $4.6 \%$ of all the words),

- Twitter (1993 words) - 56 items, i.e. $2.8 \%$ of all the words on Twitter ( 21 items used by native speakers in 899 words, i.e. $2.3 \%$ of all the words +35 used by Indian speakers in 1094 words, i.e. $3.2 \%$ of all the words),

- Facebook (fanpages) (2010 words) - 46 items, i.e. 2.2\% of all the Facebook-fanpages words analyzed ( 21 items used by native speakers in 1380 words, i.e. $1.5 \%$ of all the words used by them +25 utilized by Indian speakers in 630 words, i.e. $3.9 \%$ of all the words),

- Facebook (private) (2342 words) - 39 items, i.e. $1.7 \%$ of all the words found in the private Facebook posts analyzed ( 21 used by native speakers in 1280 words, i.e. $1.6 \%$ of all the words +18 items used by Indian speakers in 1062 words, i.e. $1.7 \%$ of all the words there).

Whereas Twitter and Facebook do not exhibit a striking difference, with, however, a certain tendency for Twitter to encourage the use of the non-standard language more (possibly due to the word limitation imposed on the posts), it is with YouTube that the users associate the use of abbreviated language forms most, and notably those of Indian background. It may be best explained by the fact that it is a medium where users often assume nicknames that hide their true identity, which is why they can feel more at liberty not to obey the usual rules of conduct, and even when their true names are provided, chances are that nobody there will know or recognize them. Although the topic of comments is the same also on Facebook-fanpages, the share of abbreviations is far lower there as far as the actual number of abbreviations is concerned, ca. a third of the YouTube use. It is assumed that the reason behind it is the fact that, contrary to the aforementioned medium, most writers use their real names when commenting on the posts, and there might be fear that they will lose face in front of some people that know them. The other end of the scale in comparison to YouTube are the private accounts of users on Facebook, the medium where all the followers and friends of a given user know who he or she is, and anything that they do online may enhance their face or, on the contrary, damage it, for if they do things against the norms of the community, they may both violate the sociality rights of the community and threaten individual face needs (cf. Spencer-Oatey 2007). This is probably why the use of unorthodox spellings appears to be rather infrequent, especially by comparison to the other sites. Interestingly enough, Twitter is also a type of an 
SNS where people know who the post authors are. However, as stated above, in the cases studied the acquaintance is probably not an intimate one, but much more of a fan admiring his/her idol from a distance. In such cases the use of spellings which do not fit the standard norms, beside other unorthodox things said or done, might be seen as an act, a way of vying for popularity, creating one's image as an original person, a trend-setter, or at best - of someone who knows the "tricks of the trade", i.e. the - often stereotypical - requirements of the medium of communication. The word limitation on Twitter might serve as an explanation too, however, it could be seen above that the share of abbreviations on Twitter is the same as on YouTube, where there are no restrictions concerning the length of posts (i.e. $2.8 \%$ of all the words found on each of the sites, respectively), this factor cannot then be viewed as most influential for Twitter.

It also needs to be observed that the highest scoring platforms, YouTube and Twitter, owe their high shares of abbreviations particularly to the Indian group of users, who exceed native speakers considerably on each platform (particularly on YouTube and Facebook-fanpages). These differences therefore appear to point to the existence of a local norm and a different, positive indexicality of abbreviations in the Indian culture in comparison to the British-American users, as was already pointed out by, among others, Pingali (2009) or Dąbrowska (2012), in both the offline and online use of English, respectively.

\section{Distribution of types of abbreviations according to the site}

The overview of the collected examples has also rendered more specific results concerning the popularity of the identified categories of abbreviations (see above) in relation to the type of SNS in which they were found. The table below provides data with regard to the sites analyzed and the categories of abbreviations used, with the most popular types in a given site highlighted (for examples of items from particular categories see the last section of the analysis):

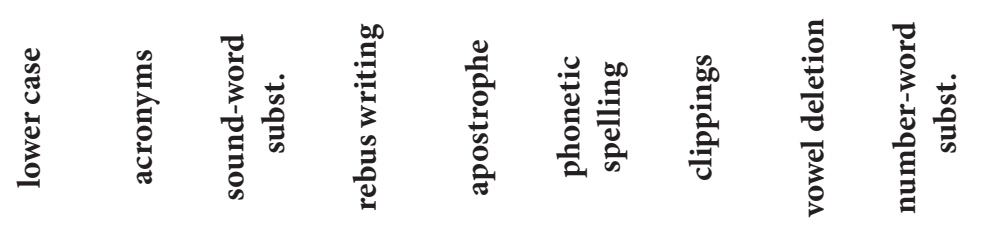

\begin{tabular}{lccccccccc}
\hline YT (110) & $\mathbf{5 0}$ & $\mathbf{3 0}$ & 6 & 4 & $\mathbf{1 2}$ & 6 & 1 & - & 1 \\
TW (56) & 2 & 7 & $\mathbf{1 6}$ & $\mathbf{1 1}$ & 4 & 2 & 5 & 6 & 3 \\
FB-FP (46) & $\mathbf{1 6}$ & $\mathbf{1 1}$ & 1 & - & 3 & $\mathbf{9}$ & 3 & 3 & - \\
FB-P (39) & 6 & 8 & $\mathbf{1 0}$ & 4 & - & 1 & 4 & 1 & -
\end{tabular}


The conclusions that the above data lead to are quite elucidating. Out of the nine categories of abbreviations identified in the material altogether some are represented by only a tiny number of examples, while others constitute a visible majority of all (cf. the use of the lower case found in $29 \%$ of all the examples with $1.6 \%$ of the numberword substitutions). What may immediately be noticed is that the number-word substitutions, like want 2, every1, i.e. the forms which are particularly striking as the CMC innovations and discussed most, especially in earlier analyses concerning the use of Netlingo (cf. Crystal 2001, 2006, 2008; Thurlow, Lengel, Tomic 2004) are hardly used, irrespective of site. Rare also are clippings, though found in each of the three SNSs, as well as vowel deletion examples. The latter, however, though represented by few examples in all, appear to be most visible on Twitter (and are absent from YouTube), which points to the fact that their use may result from the message length limitation rather than the overall fashion on CMC (which the distribution of clippings also confirms). Twitter is also in the lead as far as sound-word substitution and rebus-writing are concerned. The predomination of these here, contrary to YouTube (the platform with the greatest number of abbreviations and modifications in all, as we recall) may be caused by space limitation too, but also, and perhaps primarily, as might be assumed, by the trend-setting character of Tweets produced by celebrities (e.g. looQ, May u always shine as bright as u do!, Just want 2 thank everyone at Surya hospital 4 all $d$ wonderful work they $r$ doing \& $h v$ done 4 both my moms and evryı else as well. Hats off!). Sound-word substitutions also appear to be popular among users of Facebook in their private communication (e.g. Thank $u$ beautiful people for all ur bday wishes. I owe u a piece of cake!), which points to the general, neutral character of the type in CMC, contrary to rebus-writing, a strategy which is most time-consuming in its creation as well as decoding and requires greatest level of creativity.

While sound-word substitutions are the category that dominates on both Twitter and Facebook-private accounts, the majority of the remaining categories, viz. apostrophes, acronyms, and most notably lower case spelling are found in the first place on YouTube, and the latter two turn out to be most prominent also on Facebook-fanpages. Interestingly, there appear to be visible differences in the use of the aforementioned. The lack of apostrophes dominates only on YouTube, which can be explained quite easily as a marker of informal behaviour that challenges standard language requirements and speedy, less monitored typing. The use of acronyms, on the other hand, most frequent on both YouTube and Facebook-fanpages, stresses similarities in the use of both SNSs as a public type of communication, but at the same time a very informal one, and often taking place between people who share some in-group kind of knowledge or context of communication, otherwise the application of such acronyms as $S R K, h b, P M$ or $R T$ would not be understandable to chance users. Thus, they play the role of both making the communication smoother and faster and stress in-group identity as well. This appears to be different from the most frequent element of all on all the sites, and especially on YouTube, as indicated above (i.e. $29 \%$ of all the collected examples of abbreviations) - that of lower case spelling in words requiring the use of capital letters in the standard form. The use of 
lower case no doubt speeds up the writing, yet, as the posts on YouTube and Facebook do not have any major length limitation, and they do not need to be written as quickly as e.g. when communicating via Instant Messaging, the dominant factor behind the presence of this strategy appears to be the level of informality and tendency to ignore the standard rules. The high share of this type on YouTube in particular marks this type of register as the most informal one of the four analysed.

\section{Gender preferences}

\begin{tabular}{lccc|cccc} 
& \multicolumn{2}{c}{ Women } & \multicolumn{4}{c}{ Men } \\
& $\begin{array}{c}\text { Anglo- } \\
\text { Saxon }\end{array}$ & Indian & Total & & $\begin{array}{c}\text { Anglo- } \\
\text { Saxon }\end{array}$ & Indian & Total \\
\hline YT & 17 & $\mathbf{3 0}$ & $\mathbf{4 7}$ & YT & $\mathbf{2 7}$ & $\mathbf{3 6}$ & $\mathbf{6 3}$ \\
TW & 12 & 18 & 30 & TW & 9 & 17 & 26 \\
FB-FP & 9 & 12 & 21 & FB-FP & 12 & 13 & 25 \\
FB-P & $\mathbf{1 8}$ & 14 & 32 & FB-P & 3 & 4 & 7
\end{tabular}

An earlier study concerning the use of English on Facebook demonstrated a certain difference in the frequency of use of informal language strategies with regard to the gender of its users, hinting at the fact that it was women who chose to flout the rules of spelling more often than men (cf. Dąbrowska 2013). The overview of the collected material in respect of gender of the users in the SNSs analyzed here also pointed to some gender preferences regarding the use of abbreviations or lack thereof (see the table above with the SNSs with highest frequencies of abbreviations for the two genders marked in bold), confirming a more frequent use of nonstandard spelling among the female users. Altogether, the 280 posts ( 4831 words) written by women contained 130 items of shortenings and CMC modifications ( $3 \%$ of all the words found in women's posts), and the 280 posts ( 5364 words) written by men produced 121 of them ( $2 \%$ of all the words used by men). The difference is not huge, yet stable - when split into the Anglo-Saxon vs. Indian women and men, respectively, the ratio shows 56 abbreviations used by Anglo-Saxon women (out of 2737 words, i.e. $2 \%$ of the word count) and 51 by Anglo-Saxon men (out of 3229 , i.e. $1.5 \%$ of the word count), as well as 74 abbreviations utilized by Indian women (out of 2094 words, and thus $3.5 \%$ of all the words used by women) and 70 by Indian men (out of 2135 words, i.e. $3.2 \%$ of all). It therefore shows the same tendency for women to like the use of non-standard spellings across cultures more than is the case with men. Some classical sociolinguistic studies show that women prefer the use of standard language (cf. Trudgill 1972; Coates 1993), which would be the opposite tendency to the one hinted at here. Yet, as another investigation has demonstrated, it is also women who most often lead the process of language change, 
which means the use of informal or non-standard language use (cf. Milroy 1980). The two types of findings are only apparently contradictory. As Chambers (2003) states, women's tendency is to opt for what is more prestigious. If there is no change in progress involved, women show preference for what is more standard, and if language change is under way, women, as being more mobile and open to contacts with others tend to be in the foreground of the process. The use of language online is obviously an area which demonstrates frequent innovations compared to offline use, the observation of a greater frequency of non-standard forms of spelling in posts written by women is therefore in line with the general findings (cf. Herring, Zelenkauskaite 2008; Bieswanger 2010).

When viewed from the point of view of the four types of register analyzed here, however, the two genders seem to differ somewhat in their choices. The data in the tables demonstrate that the vast majority of the modifications used by men, both Anglo-Saxon and Indian, are located on YouTube (63 in all, i.e. $25 \%$ of all the items found, 27 , i.e. $11 \%$ produced by the native users and 36 , i.e. $14 \%$ by second-language users, respectively). While Twitter and Facebook-fanpages show an almost identical overall number (with differences, however, when split into the native and secondlanguage users), Facebook-private accounts maintained by men show a very low presence of abbreviations there ( 7 in all, i.e. $2.8 \%$ of all the items). The data found with men are not reflected in terms of their distribution with regard to women. Women (Anglo-Saxon and Indian alike) also show the most frequent use of nonstandard spelling on YouTube (47, i.e. $19 \%$ of all, so less than in the case of men, with 17 items, i.e. $7 \%$ used by native, and 30 items, i.e. $12 \%$ of all, used by Indian women, respectively). Female Twitter and Facebook-fanpage posts have similar shares of the non-standard spellings to those of men (30, i.e. $12 \%$ and 21 , i.e. $8 \%$ of items, respectively, with again a greater frequency observed in the Indian group). However, the private accounts of women on Facebook show a much higher use of abbreviations than was the case with men (32, i.e. $13 \%$ in all, split into 18 , i.e. $7 \%$ and 14 , i.e. $6 \%$ for the native and second-language users, compared to 7 , i.e. $3 \%$ altogether in the male group, with 3 items, i.e. $1 \%$ and 4 , i.e. $2 \%$ for the native and second-language users, respectively). Thus, as regards women, their private Facebook communication encourages them to use non-standard spelling far more often (the second largest group for women) than is the case with men. A similar tendency was observed on an exclusively Facebook-generated sample by native, second- and foreign-language users of English (Dąbrowska 2013). It was then concluded that women used non-standard spellings more often than men, but they used informal and slang vocabulary less frequently than men - this may then prove and corroborate the fact that women prefer to relax their language use and create an informal atmosphere by how they say/write their messages, and men - by their choice of vocabulary.

The overview of the non-standard spellings of English on the analyzed SNSs used by both native and second language users also indicates certain cultural regularities. In total, it is the Indian users that dominated as far as the use of the greatest number of non-standard forms, using 144 , i.e. $57 \%$ of them in all (women - 74 , i.e. $29 \%$, men -7 o, i.e. $28 \%$ ), as opposed to the native users, who made use of 107 , 


\begin{tabular}{lcccc} 
& $\begin{array}{c}\text { A-S } \\
\text { women }\end{array}$ & $\begin{array}{c}\text { Indian } \\
\text { women }\end{array}$ & A-S men & $\begin{array}{c}\text { Indian } \\
\text { men }\end{array}$ \\
\hline Lower case (74) & 15 & $\mathbf{2 2}$ & 13 & $\mathbf{2 4}$ \\
Acronyms (56) & $\mathbf{1 9}$ & 9 & 13 & $\mathbf{1 5}$ \\
Sound-word substitution (33) & 5 & 13 & 4 & 11 \\
Rebus writing (23) & 8 & 6 & 5 & 4 \\
Apostrophe deletion (18) & 3 & 4 & 8 & 3 \\
Phonetic spelling (18) & 1 & 9 & 2 & 6 \\
Clippings (13) & 2 & 5 & 4 & 2 \\
Vowel deletion (10) & - & 4 & 2 & 4 \\
Number-word substitution (4) & 2 & 1 & - & 1
\end{tabular}

Distribution of items according to the culture and language status

i.e. $43 \%$ of such forms in all ( 56 , i.e. $22 \%$ used by women, 51 , i.e. $21 \%$ by men). A similar tendency to use abbreviations, notably those based on vowel deletion, was already identified in the analysis of Indian English on Facebook (cf. Dąbrowska 2012). It was then linked with the fact that in the Devanagari script used for writing Hindi (the dominant language of all the respondents whose posts were investigated) the short vowel /a/ is an obligatory element of interpretation of every Devanagari character, it is therefore not indicated by any additional element in writing. This tendency to assume the existence of a vowel, here by default of any vowel, as it seems, may then be transferred onto the use of English in writing as well. However, in the material analyzed here vowel deletion was only marginally visible (10 items), but still with a preference for it in the Indian group (8 items). What dominated by far in the forms used in both native and second language messages (with one tiny exception for native women) was the use of lower case. Yet, it was again Indian women and men, respectively, who made use of it a lot more than native speakers $(22$, i.e. $9 \%$ of all the items in the case of Indian women and 24 , i.e. $10 \%$ in the case of Indian men vs. 15 , i.e. $6 \%$ in the case of native women and 13 , i.e. $5 \%$ in the case of native men). This discrepancy might be explained not only by the overall popularity of this form of relaxing spelling online without at the same time making the forms difficult to decode, but in the case of Indian users possibly also due to the influence of the Devanagari script, in which there is no distinction between capital and lower case characters.

One more category, the sound-word substitution, also points clearly to its popularity among Indian users. While it might be linked with the fact that as non-native users Indians might be more willing to follow the phonetic spelling of words than the native ones, a possible reason here might be simply the CMC fashion to write 
certain words, notably pronouns like $u$ or auxiliary verbs like $r$ in a non-standard, but by now a well-established online form. This appears to be corroborated by another subcategory which shows Indian users' preference for it, i.e. the phonetic spelling modifications proper, which do not necessarily substitute whole words or syllables, but individual sounds (e.g. lil, muvi). The exposure to traditional spelling from early childhood might, in turn, discourage native users from such a frequent choice of this option.

On the other hand, a glance at the table above shows that apostrophe deletion and, especially, rebus writing seem to tip the scales towards the native users more than the second-language English speakers. Apostrophe deletion is one of those modifications which might lead to misunderstandings of the forms used (e.g. its vs. it's or moms vs. mom's), which is why non-native users might be more inclined to keep the spelling right, while native ones, and as can be seen, men in particular, seem to feel more in a position to introduce modifications as rightful "owners" of English. This may also be the case with rebus writing, where modifications create forms far more remote from the original spelling. The use of this type of abbreviation requires not only language creativity, but also knowledge of the original forms of words as well as sound- and number-word correspondences, which is why native speakers may feel bolder to undertake such more far-fetched alterations than non-native ones.

\section{The character of the non-standard spelling}

The final aspect to be looked into in our overview is the actual analysis of the types of abbreviations used. As could be gathered from the above tables, there is an obvious numerical diversity concerning the representation of the identified categories, ranging between the lower case forms, with as many as 74 examples (29\%), and acronyms with $56(22 \%)$ at one end, and barely 4 (1.5\%) examples of number-word substitution at the other. The categories in between include, respectively, soundword substitutions - $33(13 \%)$, rebus writing - 23 (9\%), apostrophe deletion - 18 ( $7 \%$ ), phonetic spelling - 18 (7\%), clippings - 13 (5\%) and vowel deletion - $10(4 \%)$. The above can be illustrated by the following examples:

- Lower case - proper names (e.g. kaira, shah, emma stone, trump), titles (fan, la la land, moonlight), pronouns, e.g. $i$

- Acronyms - emotive markers, e.g. omg, lol, wtf, lmao(ooo), smh, kys, interjections, e.g. btw, imo, fyi, ICYMI, set phrases, e.g. wishes - hny, hbd, hb, or Internet activities, e.g. RT, PM, taboo meanings, e.g. BFFs, $P O S$, names and titles - SRK, FB

- Sound-word substitution - $u, u r, y, r$

- Rebus writing - bcoz, looQ, plsss, than $x x x x, f^{\prime} d u p, x x x, r 8,2 n i g h t^{2}$

$2 \quad r 8$ and 2night have been classified as instances of rebus writing and not as number-word substitutions on the grounds that the figures used in them do not correspond to separate words (elements of compounds), but to a sound and a prefix, respectively. 
- Apostrophe deletion - auxiliary verbs, e.g. its, dont, didnt, aint, im; Saxon Genitive - moms, warrens; other contractions, e.g. lets

- Phonetic spelling - lil, dat, muvi, luv, coz, happieee buddayyy, awtstanding

- Clippings - congrats, fab, pic, tho, bros, subs

- Vowel deletion - $h v, n x t, p l s, n d, f r$

- Number-word substitution - 2 wear, want 2, every1, done 4

The overview of the above allows us to conclude that the examples can be divided into two broad subcategories. On the one hand, there are one-off items marked by a simple formal modification, and these are primarily lower case spellings, the largest of the categories, and to some extent also phonetic spellings and vowel deletions. Some examples of the apostrophe deletions and rebus writing also belong to this type. In their case, once the user knows the method of their formation, their decoding is straightforward. On the other hand, clippings, sound-word substitutions, and most of all acronyms are a relatively limited group of forms which, if they are not familiar to the users, may lead to a complete breakdown in communication, and as such they have to be learnt in order to be used in a meaningful way. I see the latter, therefore, as an enrichment of the English lexicon, not only an online spelling modification, and these are the forms, especially acronyms, that have a chance to acquire a fairly mandatory use online and also survive or even spread in the offline world as new vocabulary items (rather than phrases), though at times with the pronunciation very distant from their written form.

\section{Concluding remarks}

As a result of the above analysis of abbreviations and spelling modifications used on the three sites, and four types of register, with regard to their popularity and character as well as the users' gender and the language status the following observations can be drawn:

- as assumed, the frequency of the use of abbreviated, non-standard language online on the investigated SNSs is relatively infrequent, with the overall ratio of the use around 0.45 per post (0.78 on YouTube, 0.4 on Twitter, 0.33 on Facebookfanpages, and 0.28 on Facebook-private accounts),

- the type of the communication platform has a bearing on the frequency of the abbreviations used - YouTube encourages the use of such modifications most, possibly due to anonymity of the users, and Facebook friend network, where the person's image and smooth communication are more at stake - the least,

- the greatest difference in the frequency of Netlingo use has been found in connection with the cultural (native language vs. second language) background of SNSs users, with the Indian users showing a considerably greater preference for the abbreviated forms than the native speakers, thereby confirming the different local practice regarding the analyzed code, 
- there appears to be a slight but visible preference for the use of abbreviated forms on the part of women in both cultures investigated (with the ratio of 130 forms used by women to 121 by men in all),

- there is a more pronounced gender preference for the use of shortenings as regards the type of SNS, with women, notably of Anglo-Saxon background, more often opting for their use in private communication on Facebook, and men of both cultures - on YouTube. While Indian women also follow the fashion of using abbreviations most on YouTube, men in both cultures avoid using Netlingo on Facebook to a considerable extent. Thus, using abbreviated and non-standard forms of language may be treated as an in-group marker for women in private Facebook network (as a way of creating friendly relations and relaxed atmosphere),

- YouTube and Facebook-fanpages users appear to favour the use of lower case, acronyms, and apostrophe omission as regards their choice of the abbreviated language, the possible reason for which may be the tendency to opt for relaxed, informal writing that defies the norms of language use in a (fully or partly) anonymous setting, with emotional displays in the foreground,

- on Twitter and Facebook private profiles, where users are known to their friends or followers, the preferred strategies tend to be the sound-word substitutions and rebus writing. The former appear to act as community of practice markers and they do not obstruct personal communication, while the rebus forms on Twitter may help the Tweeps to build their public image as "cool" and technologically savvy Internet users.

To sum up, the use of abbreviations online appears to be medium-sensitive and reflects variation in terms of the users' culture and gender. Their use, however, is not pervasive and omnipresent, contrary to the academic accounts indicated earlier, and shows inconsistency of application. This might also hint at a possible decline in the frequency of use of abbreviations online, a topic worth separate investigation. The likely forms that may acquire a more stable status in informal online (and subsequently also offline) communication are especially acronyms. They tend to fulfil the role of emotive discourse markers and interjections, whereby they can expand on the informal English lexicon, for they act as single units of meaning, without at the same time hindering the decoding of the remaining part of the message.

\section{References}

Agha A. 2005. Voice, footing, enregisterment. - Journal of Linguistic Anthropology 15: 38-59. Anis J. 2007. Neography. Unconventional spelling in French SMS text messages. - Danet B., Herring S.C. (eds.). The multilingual Internet. Language, culture and communication online. Oxford: 87-115.

Baron N. 2008. Always on. Language in an online and mobile world. Oxford. 
Bieswanger M. 2007. 2 abbrevi8 or not 2 abbrevi8: A contrastive analysis of different spaceand time-saving strategies in English and German text messages. - Texas Linguistic Forum 50. [Retrieved from http://studentorgs.utexas.edu/salsa/proceedings/2006/ Bieswanger.pdf].

Bieswanger M. 2010. Gendered language use in computer-mediated communication. Typography in text messaging. - Bieswanger M., Motschenbacher H., Mühleisen S. (eds.). Language in its socio-cultural context. New explorations in gendered, global and media uses. Frankfurt am Main: 157-172.

Blommaert J. 2010. The sociolinguistics of globalization. Cambridge.

boyd d. 2008. Why youth <3 social network sites: The role of networked publics in teenaged social life. - Buckingham D. (ed.). Youth, identity and digital media. Cambridge: 119-142.

Chambers J.K. 2003. Sociolinguistic theory. [2 $2^{\text {nd }}$ edition]. Oxford.

Coates J. 1993. Women, men and language. [2 ${ }^{\text {nd }}$ edition]. London, New York.

Coetzee F. 2012. The multilingual literacy practices of residents living a coloured, Afrikaansdominant neighbourhoods in Cape Town: A sociolinguistic study. [Unpublished MA thesis, University of Cape Town].

Crystal D. 2001. Language and the Internet. Cambridge

Crystal D. 2006. Language and the Internet. [2 ${ }^{\text {nd }}$ edition]. Cambridge.

Crystal D. 2008. Txtng. The gr8 db8. Oxford.

Crystal D. 2011. Internet linguistics: A student guide. London, New York.

Crystal D. 2013. Spell it out. The singular story of English spelling. London.

Dąbrowska M. 2011. Language economy in short text messages. - Studia Linguistica Universitatis Iagellonicae Cracoviensis 128: 7-21.

Dąbrowska M. 2012. 'Happy b'day bhaiya.' Characteristics of Facebook Indian English. Studia Linguistica Universitatis Iagellonicae Cracoviensis 129: 61-89.

Dąbrowska M. 2013. Variation in language. Faces of Facebook English. Frankfurt am Main.

Danesi M. 2016. Language, society and new media. Sociolinguistics today. New York, London.

Deumert A. 2014. Sociolinguistics and mobile communication. Edinburgh.

Deumert A., Lexander K.V. 2013. Texting Africa: Writing as performance. - Journal of Sociolinguistics 17: 522-546.

Freeborn D. 1998. From Old English to Standard English. A course book in language variation across time. [ $2^{\text {nd }}$ edition]. Houndmills, Basingstoke.

Graddol D. 2006. English next. Why global English may mean the end of "English as a Foreign Language." London.

Herring S., Zelenkauskaite A. 2008. Gendered typography: Abbreviation and insertion in Italian iTV SMS. - Siegel J.F., Nagle T.C., Lorente-Lapole A., Auger J. (eds.). Gender in language: Classic questions, new contexts. Bloomington (IN): 73-92.

Hinnenkamp V. 2008. Deutsch, Doyc or Doitsch? Chatters as languagers - The case of a German-Turkish chat room. - International Journal of Multilingualism 5: 253-275.

Ling R. 2005. The socio-linguistics of SMS: An analysis of SMS use by a random sample of Norwegians. - Ling R., Pedersen P.E. (eds.). Mobile communications: Renegotiation of the social sphere. London: 335-349.

McIntosh J. 2010. Mobile phones and Mipoho's prophecy: The powers and dangers of flying language. - American Ethnologist 37: 337-353.

Milroy L. 1980. Language and social networks. London, Baltimore.

Palfreyman D., Al Khalil M. 2007. 'A funky language for teenz to use': Representing a Gulf Arabic in instant messaging. - Danet B., Herring S.C. (eds.). The multilingual Internet. Language, culture and communication online. Oxford: 43-63. 
Pennycook A. 2007. Global Englishes and transcultural flows. London.

Pingali S. 2009. Indian English. Edinburgh.

Saenger P. 2010. The impact of the early printed page on the history of reading. - Gadd I. (ed.). The history of the book in the West. [vol. 2: 1455-1700]. Surrey (UK): 385-449.

Spencer-Oatey H. 2007. Theories of identity and the analysis of face. - Journal of Pragmatics 39: 639-656.

Squires L. 2010. Enregistering Internet language. - Language in Society 172: 51-77.

Stevenson J. 2000. The language of Internet Relay Chat. [Retrieved from http://www.demo. inty.net/Units/Internet\%20Relay\%20Chat.htm].

Su H-Y. 2007. The multilingual and multiorthographic Taiwan-based Internet: Creative uses of writing systems on college-affiliated BBSs. - Danet B., Herring S.C. (eds.). The multilingual Internet. Language, culture and communication online. Oxford: 64-86.

Tagg C. 2009. A corpus linguistic study of SMS text messaging. [Unpublished PhD thesis, University of Birmingham].

Tagliamonte S.A., Denis D. 2008. Linguistic ruin? Lol! Instant messaging and teen language. American Speech 83: 3-34.

Thurlow C., Lengel L., Tomic A. 2004. Computer mediated communication. Social interaction and the Internet. London.

Trudgill P. 1972. Sex, covert prestige, and linguistic change in the urban British English of Norwich. - Language in Society 1: 179-196. 